\title{
The Effects of Dizocilpine and Phencyclidine on Prepulse Inhibition of the Acoustic Startle Reflex and on Prepulse-Elicited Reactivity in C57BL6 Mice
}

\author{
Benjamin K Yee*,', DL Tilly Chang' and Joram Feldon' \\ 'Laboratory of Behavioural Neurobiology, Swiss Federal Institute of Technology Zurich, Schwerzenbach, Switzerland
}

\begin{abstract}
Prepulse inhibition (PPI) of the acoustic startle response refers to the reduction in startle reaction to a startle-eliciting stimulus when it is shortly preceded by a subthreshold prepulse stimulus. Here, we evaluated the possible effects on prepulse-elicited reactivity by dizocilpine (MK-80I) and phencyclidine (PCP) in the PPI of acoustic startle paradigm in C57BL6/J mice. The aim was to ascertain whether these two drugs would affect prepulse-elicited reactivity in a manner similar to apomorphine, which enhances prepulse-elicited reactivity at doses that disrupt PPI. In two dose-response studies, we showed that both drugs exhibited a tendency to attenuate prepulse-elicited reaction at higher doses when PPI was severely disrupted. On the other hand, at lower doses when PPI was marginally disrupted, reaction to the prepulse, if anything, tended to increase. It is concluded that PPI disruption induced by noncompetitive NMDA receptor antagonists can be distinguished from apomorphine-induced PPI disruption by their concomitant effects on prepulse-elicited reactivity. Our data support the suggestion that dopamine receptor agonists and NMDA receptor antagonists disrupt PPI via interference with distinct neural pathways or neuronal systems.

Neuropsychopharmacology (2004) 29, I865-1877, advance online publication, 5 May 2004; doi: I 0. I038/sj.npp. 1300480
\end{abstract}

Keywords: MK-80I; NMDA antagonist; PCP; PPI; sensorimotor gating; schizophrenia

\section{INTRODUCTION}

Prepulse inhibition (PPI) of the acoustic startle reflex refers to the reduction of the reaction to a startle-eliciting 'pulse' stimulus when it is shortly preceded by a weak 'prepulse' stimulus (Hoffman and Searle, 1965). One view posits that the prepulse impairs the reflexive motor response to the subsequent pulse stimulus as a result of reduced processing of the pulse stimulus. This effect of the prepulse upon pulse processing is commonly considered as a form of sensorimotor gating, and it can be readily demonstrated across species, from mice to humans (Braff et al, 2001; Geyer et al, 2002).

In humans, deficits in PPI have been reported in schizophrenia and schizotypal personality disorders (Braff et al, 2001). Although PPI deficits are not unique to schizophrenia and are also noted in conditions such as obsessive-compulsive disorder and Huntington's disease

\footnotetext{
*Correspondence: Dr BK Yee, Laboratory of Behavioural Neurobiology, Swiss Federal Institute of Technology Zurich, Schorenstrasse 16, CH-8603 Schwerzenbach, Switzerland, Tel: +4I I 6557417, Fax: +4I I 6557203, E-mail: benjamin.yee@behav.biol.ethz.ch

Received 20 November 2003; revised 22 March 2004; accepted 8 April 2004

Online publication: 8 April 2004 at http://www.acnp.org/citations/ Npp04080403535/default.pdf
}

(Swerdlow et al, 1995), the relevance of PPI and its disruption to schizophrenia has been of particular interest because the expression of PPI can be influenced by dopaminergic transmission (see Geyer et al, 2001, 2002), and the dopamine hypothesis of schizophrenia has remained highly influential (Snyder, 1976). Pharmacologically induced disruption of PPI has thus been employed as an animal model of schizophrenia-like sensorimotor gating deficits (Swerdlow et al, 2000).

PPI is reliably disrupted by dopamine agonists, such as apomorphine in both rats and mice (eg Martinez et al, 2000; Varty et al, 2001). In addition, noncompetitive NMDA ( $N$ methyl-D-aspartate) receptor antagonists, such as dizocilpine (MK-801) and phencyclidine (PCP), also disrupt PPI (Curzon and Decker, 1998). Antagonism against the disruptive effect on PPI by these drugs in rodents has often been taken as evidence of potential antipsychotic properties. In the mouse, an increasing number of targeted molecular manipulations are also reported to affect the expression of PPI (see Geyer et al, 2002). However, little attempts have been made to ascertain whether the disruptions of PPI induced by pharmacologically distinct classes of drugs or various specific targeted mutations can all be readily understood as identical, that is, being attributed to the same disturbance to a common psychological function. The possibility that this may not be the case would bear 
interesting implications over the fact that PPI disruption is identifiable across distinct neuropsychiatric conditions (see Swerdlow et al, 1995).

Recently, we have attempted to assess one hypothesis concerning the psychophysical mechanism underlying apomorphine-induced PPI disruption originally put forward by Davis et al (1990). These authors suggested that apomorphine effectively attenuates the detectability of the prepulse stimulus, and thereby reduces the negative impact of the prepulse upon pulse stimulus processing. However, we have failed to confirm their hypothesis in an experiment in which we conducted a detailed analysis of prepulseelicited reactivity, which may be considered as an indirect measure of the detection and/or processing of the prepulse stimulus. We observed that apomorphine tended to enhance rather than to attenuate reactivity to the prepulse stimulus (Yee et al, 2004). Although it is unlikely that the observed effect of apomorphine on prepulse-elicited reactivity is itself directly responsible for the concomitant disruption of PPI, this novel observation may shed light on the neuropsychopharmacology of apomorphine as to how it may modulate the expression of PPI (see Yee et al, 2004). One clear possibility is to examine the generality of our initial finding obtained in apomorphine by testing the possible effects on prepulse-elicited reactivity by other pharmacological interventions known to disrupt PPI. However, it is unfortunate that previous studies have paid scant attention to the data obtained on trials in which only the prepulse stimulus is presented. Some recent studies have even dropped such control trials altogether (eg Martinez et al, 2000; Geyer and Swerdlow, 1998).

Here, we evaluated the possible effects on prepulseelicited reactivity by dizocilpine and PCP in the PPI of the acoustic startle paradigm. Both drugs are known to disrupt PPI in rats as well as in mice, and the aim was to ascertain whether these two drugs would also be associated with any observable effects on prepulse-elicited reactivity in a similar manner to that of apomorphine (Yee et al, 2004), and, if not, whether the two NMDA antagonists behave similarly to each other in terms of their potential effects on prepulseelicited reactivity. In the present study, the two drugs were studied in two separate dose-response experiments conducted in C57BL6/J mice. Both drugs were expected to disrupt PPI in a dose-dependent manner, and we have selected a sufficiently wide range of doses to span from doses that would be marginally effective in disrupting PPI to doses that would be highly effective. This approach allows a direct comparison between the drugs' dose-dependent effects on PPI and prepulse-elicited reactivity, and thereby to facilitate the detection of a potential dissociation between the two effects.

\section{MATERIALS AND METHODS}

\section{Subjects}

The subjects consisted of male C57BL6/J mice, obtained from the SPF (specific-pathogen-free) facility in the Laboratory of Behavioural Neurobiology (Swiss Federal Institute of Technology Zurich). At the beginning of the experiment, the mice were about 4 months old and weighed 24-34 g. All animals were maintained under ad lib food and water throughout the experiment. They were housed singly in a temperature and humidity-controlled $\left(22^{\circ} \mathrm{C}, \sim 55 \%\right)$ animal facility under a reversed light-dark cycle (lights on 1900-0700 h). Behavioural testing was carried out during the dark phase of the cycle. All experiments were conducted in accordance with the National Institutes of Health Guide for Care and Use of Laboratory Animals, and were approved by the Cantonal Veterinarian's Office of Zurich.

\section{Drugs}

Dizocilpine (MK-801; obtained from Merck, Sharp \& Dohme, UK) and PCP (phencyclidine; obtained as a gift from Hoffman-La Roche, Basel, Switzerland) were dissolved in isotonic $0.9 \% \mathrm{NaCl}$ solution to achieve the desired concentration for injection. All solutions were freshly prepared on the day of testing. Dizocilpine and PCP were administered via the intraperitoneal (i.p.) route $10 \mathrm{~min}$ before testing. The volume of injection was $5 \mathrm{ml} / \mathrm{kg}$.

In Experiment 1, five doses of dizocilpine were included: $0.1,0.3,0.5,1.0$, and $3.0 \mathrm{mg} / \mathrm{kg} \quad(n=7,10,10,7,6$, respectively), in addition to a saline-injected control group $(n=9)$. In Experiment 2, five doses of PCP were included: $2.5,5.0,7.5,10$, and $15 \mathrm{mg} / \mathrm{kg}(n=8,8,8,8,9$, respectively), saline-injected subjects again constituted the control group $(n=7)$. In each experiment, mice originated from the same litter were, as far as possible, assigned to different treatment conditions in order to minimize potential confounds resulting from litter effects (Zorrilla, 1997).

\section{Apparatus}

The apparatus consisted of four acoustic startle chambers for mice (SR-LAB, San Diego Instruments, San Diego, CA, USA). Each startle chamber comprised a nonrestrictive cylindrical enclosure made of clear Plexiglas attached horizontally on a mobile platform, which in turn was resting on a solid base inside a sound-attenuated isolation cubicle. A high-frequency loudspeaker mounted directly above the animal enclosure inside each cubicle produced a continuous background noise of $65 \mathrm{~dB}$ (A-scale) and the various acoustic stimuli in the form of white noise. The pulse and prepulse stimuli employed were in the form of sudden elevation in noise level (sustaining for 40 and $20 \mathrm{~ms}$, respectively) from the background, with a rise time of $0.2-$ $1.0 \mathrm{~ms}$. Vibrations of the Plexiglas enclosure caused by the whole-body startle response of the animal were converted into analogue signals by a piezoelectric unit attached to the platform. These signals were digitized and stored by a computer. A total of 130 readings were taken at $0.5-\mathrm{ms}$ intervals (ie spanning across $65 \mathrm{~ms}$ ), starting at the onset of the startle stimulus in pulse-alone and prepulse-plus-pulse trials, and at the onset of the prepulse stimulus in prepulsealone trials. The average amplitude (in arbitrary units) over the $65 \mathrm{~ms}$ was used to determine the stimulus reactivity. The sensitivity of the stabilimeter was regularly calibrated to ensure consistency between chambers and across sessions.

\section{Procedures}

In the demonstration of prepulse inhibition (PPI) of the acoustic startle reflex, subjects were presented with a series 
of discrete trials comprising a mixture of four types of trials. These included pulse-alone trials, prepulseplus-pulse trials, prepulse-alone trials, and trials in which no discrete stimulus, other than the constant background noise, was presented (denoted here as 'no-stimulus' trials). A reduction of startle magnitude in prepulseplus-pulse trials relative to those in pulse-alone trials constitutes PPI. The pulse stimulus employed was $120 \mathrm{~dB}_{\mathrm{A}}$ in intensity and $40 \mathrm{~ms}$ in duration. Prepulses of various intensities were employed: $69,73,77,81$, and $85 \mathrm{~dB}_{\mathrm{A}}$, which corresponded to $4,8,12,16$, and $20 \mathrm{~dB}_{\mathrm{A}}$ above background, respectively. The duration of prepulse stimuli was $20 \mathrm{~ms}$. The SOA (stimulus onset asynchrony) of the prepulse and pulse stimuli on prepulse-plus-pulse trials was $100 \mathrm{~ms}$.

A session began with the animals being placed into the Plexiglas enclosure. They were acclimatized to the apparatus for 2 min before the first trial began. The first six trials consisted of startle-alone trials, which served to habituate and stabilize the animals' startle response. Subsequently, the animals were presented with 12 blocks of discrete test trials. Each block consisted of one trial of each of the following trial types: pulse-alone, prepulse-plus-pulse trials of each of the five levels of prepulse, prepulse-alone of each of the five levels of prepulse, and no stimulus (ie background alone). The session was concluded with the final block of six consecutive startle-alone trials. The interval between successive trials was variable with a mean of $15 \mathrm{~s}$ (ranging from 10 to $20 \mathrm{~s}$ ).

\section{Terminology, Derivation, and Analysis of Dependent Measures}

The output of the stabilimeter that measured the whole body motion of the subject, within the pre-defined response window, on each and every trial in the test session constituted the raw data. This output was referred to as the reactivity score, and was expressed in arbitrary units. As detailed below, the data were categorized into different subsets according to their relevance to distinct behavioral constructs and were separately analyzed.

Startle habituation. To gauge the effect of startle habituation, we compared the mean reactivity obtained in the first block against the last block of six consecutive pulse-alone trials. This entailed a $6 \times 2$ (treatment $\times 6$-trial blocks) splitplot ANOVA. A natural logarithmic transformation was carried out on the mean reactivity scores before the data were submitted to analysis in order to better conform to the normality and variance homogeneity assumptions of parametric ANOVA.

Startle reactivity. The mean reactivity score obtained on the middle 12 pulse-alone trials was analyzed in order to evaluate startle reactivity, a term which we reserved to describe this measure. This entailed a one-way ANOVA evaluating difference among treatment conditions (vehicle and increasing doses of the relevant drug). A natural logarithmic transformation was also carried out on the mean reactivity score before the data were submitted to analysis.
Prepulse inhibition (\%PPI). The reactivity scores obtained on the middle 12 pulse-alone trials and prepulse-plus-pulse trials were utilized to evaluate PPI. To measure or index PPI, it is customary to normalize the reduction of startle reaction (ie reactivity score obtained on prepulse-plus-pulse trials at different prepulse intensities) with respect to each subject's average reactivity score across the 12 pulse-alone trials, and express as percent inhibition. The individual mean startle reactivity thus serves as the baseline against which the effectiveness of the prepulse to inhibit the startle response to the subsequent pulse stimulus is expressed in percent, that is, $\% P P I=($ (pulsealone-prepulse-plus-pulse) $\div$ pulse-alone $\times 100 \%$ ).

The measure of $\%$ PPI was subjected to a $6 \times 5$ (treatment $\times$ prepulse intensities) split-plot ANOVA. The withinsubjects factor prepulse intensities consisted of five levels, referring to prepulse intensities $+4,+8,+12,+16$, and $+20 \mathrm{~dB}_{\mathrm{A}}$ above background.

The main effect of treatment as well as its interaction with the within-subject factor of prepulse intensities are indicative of the differential expression of PPI between treatment conditions.

Prepulse inhibition (the rate of startle reduction as a function of increasing prepulse intensity). This alternative approach of PPI assessment has been proposed by Swerdlow et al (2000) as a necessary supplement to the $\%$ PPI analysis when a clear difference in startle reactivity (ie pulse-alone trials) exists between treatment conditions. In this approach, the PPI effect is gauged without the adoption of a percentage measure. The same data set consisting of the reactivity scores obtained on the middle 12 pulse-alone trials and prepulse-plus-pulse trials can be directly compared using a $6 \times 6$ (treatment $\times$ prepulse intensities) splitplot ANOVA.

This entailed the use of a within-subjects factor consisting of six levels, denoting the five prepulse-plus-pulse trialtypes (prepulse intensities $=+4,+8,+12,+16$, $+20 \mathrm{~dB}_{\mathrm{A}}$ above background) and the pulse-alone trial, which can be considered here as having a prepulse of $+0 \mathrm{~dB}_{\mathrm{A}}$ above background. In this analysis, only the interaction between the prepulse intensities factor and the treatment factor is relevant to the comparison of PPI expression between treatment conditions, because the main effect of treatment would also be subjected to systematic change in startle reactivity as such, regardless of prepulse intensities.

Again, a natural logarithmic transformation was carried out before the data were submitted to analysis, in order to better conform to the normality and variance homogeneity assumption of parametric ANOVA.

Prepulse-elicited reactivity. To obtain a measure of prepulse-elicited reactivity, we included data obtained on prepulse-alone trials as well as on no-stimulus trials. In the control no-stimulus trials, no sudden stimulus above the constant background was presented. A comparison between prepulse-alone trials at each level of intensity and the control no-stimulus trials allowed the specific assessment of prepulse-elicited reactivity. We adopted a normalization approach similar in principle to that used for the 
conventional analysis of PPI, except that we chose to express prepulse-elicited reactivity using a difference score instead of percentages, because the low scores on nostimulus trials could lead to exaggerated percent scores and thereby led to inflated variability. To enhance the resultant data distribution, we calculated the difference score after logarithmic transformation of the individual mean reactivity score on each of the six trial types (no-stimulus and prepulse-alone trials across the five prepulse intensities).

The data were then submitted to a $6 \times 5$ (treatment $\times$ prepulse intensities) split-plot ANOVA. The main treatment effect, as well as its interaction with the within-subject factor of prepulse intensities, would be of relevance to the assessment of any differential expression of prepulse-elicited reactivity between treatment conditions.

Reactivity on no-stimulus trials. Separate analysis of reactivity on no-stimulus trials. This might reflect an effect of the treatment on spontaneous whole body movement. We previously observed such an effect with amphetamine $(2.5 \mathrm{mg} / \mathrm{kg}$, i.p. $)$, but not with apomorphine $(2.0 \mathrm{mg} / \mathrm{kg}$ s.c.), in C57BL6 mice (Yee et al, 2004).

All statistical analyses were carried out using SPSS (version 11) implemented on a PC running the Windows XP operating system. Data derived from Experiments 1 and 2 were separately analyzed, as they were completely independent. All transformations applied were established to be effective in achieving the desired effects in improving the data distribution. Even though parametric ANOVA can tolerate deviations from the normality assumption, enhanced compliance to it can improve the power of the statistical tests. Previous examination of untransformed data further confirmed that the patterns of results were largely in agreement with that obtained using the transformed data.

Fisher's LSD post hoc pair-wise comparison was used to examine the patterns of significant treatment effects. Specific analyses of covariance (ANCOVA) were conducted to examine the extent to which a significant effect emerging from an ANOVA of one variable could be explained by another variable. The $1 n$-transformed mean startle reactivity (of the middle 12 pulse-alone trials) was used as a covariate in the analysis of \%PPI and of prepulse-elicited reactivity. In addition, the mean reactivity score (ln-transformed) derived from no-stimulus trials was also used as a covariate in another ANCOVA of prepulse-elicited reactivity.

Bivariate linear correlative analyses were used to evaluate the possible relationship between the size of prepulse inhibition and the magnitude of prepulse-elicited reactivity. The possibility that such a correlation might be mediated by between-subjects variability of startle reactivity (of the middle 12 pulse-alone trials) was further evaluated using partial correlation.

\section{RESULTS}

\section{Experiment 1: Effects of Dizocilpine on PPI and Prepulse-Elicited Reactivity}

Startle habituation. There was an overall presence of startle habituation as reflected by the significant overall reduction of startle reactivity from the first to the last block of six pulse-alone trials $(\mathrm{F}(1,43)=6.71, p<0.05)$. However, a clear trend of habituation was only readily detected in the MK$801 / 1 \mathrm{mg}$ and MK-801/3 mg groups (Table 1). This led to the emergence of a significant interaction between treatment and blocks $(\mathrm{F}(5,43)=7.04, p<0.001)$. The overall level of startle reactivity also differed among treatment groups $(\mathrm{F}(5,43)=7.26, p<0.001)$. Post hoc Fisher's LSD tests indicated that dizocilpine significantly enhanced reactivity at doses of 0.3 and $0.5 \mathrm{mg} / \mathrm{kg}$, but reduced startle reactivity at $3 \mathrm{mg} / \mathrm{kg}$ relative to saline controls (all $p$ 's $<0.05$ ).

Startle reactivity. One-way ANOVA of the mean reactivity in the middle 12 pulse-alone trials yielded results similar to the overall treatment effects described above (see Table 1). There was a main effect of treatment $(\mathrm{F}(5,43)=12.97$, $p<0.001)$. Post hoc pair-wise comparisons against the saline control group again indicated that dizocilpine significantly enhanced reactivity at doses of 0.3 and $0.5 \mathrm{mg} / \mathrm{kg}$, but reduced startle reactivity at $3 \mathrm{mg} / \mathrm{kg}$ (all $p^{\prime}$ s $<0.01$ ).

\section{Prepulse inhibition.}

$\% P P I:$ It was apparent that the presence of a prepulse stimulus prior to the pulse stimulus attenuated the response magnitude to the latter. The more intense the prepulse, the stronger was the prepulse inhibition effect (Figure 1a). This gave rise to the significant main effect of prepulse intensity in the analysis of percent PPI $(\mathrm{F}(4,172)=109.38, p<0.001)$.

Dizocilpine disrupted PPI in a clear dose-dependent manner (Figure 1d). The main effect of treatment was highly significant $(\mathrm{F}(5,43)=25.22, p<0.001)$, and so was the interaction between treatment and prepulses $(\mathrm{F}(20,172)=2.03, p<0.01)$ (Figure 1a). Post hoc comparisons showed that dizocilpine, except at the lowest dose examined here $(0.1 \mathrm{mg} / \mathrm{kg})$, significantly reduced the PPI effect in comparison to the saline control group (all $p^{\prime} s<0.001$ ) (see Figure 1d).

Table I Effects of Dizocilpine (MK-80I) on Startle Habituation and Startle Reactivity

\begin{tabular}{|c|c|c|c|c|}
\hline \multirow[b]{2}{*}{ MK80I } & \multirow[b]{2}{*}{$\begin{array}{c}\text { Effect } \\
\text { relative } \\
\text { to } \\
\text { saline }\end{array}$} & \multicolumn{3}{|c|}{ Mean startle reactivity (In-transformed) } \\
\hline & & $\begin{array}{c}\text { First six } \\
\text { pulse-alone } \\
\text { (consecutive) } \\
\text { trials }\end{array}$ & $\begin{array}{c}\text { Last six } \\
\text { pulse-alone } \\
\text { (consecutive) } \\
\text { trials }\end{array}$ & $\begin{array}{c}\text { Middle I2 } \\
\text { pulse-alone } \\
\text { (distributed) } \\
\text { trials }\end{array}$ \\
\hline Saline & & $4.393 \pm 0.231$ & $4.352 \pm 0.229$ & $4.388 \pm 0.224$ \\
\hline $0.1 \mathrm{mg}$ & & $4.512 \pm 0.168$ & $4.758 \pm 0.163$ & $4.591 \pm 0.148$ \\
\hline $0.3 \mathrm{mg}$ & $\uparrow$ & $4.833 \pm 0.104$ & $5.214 \pm 0.158$ & $5.216 \pm 0.091$ \\
\hline $0.5 \mathrm{mg}$ & $\uparrow$ & $4.940 \pm 0.221$ & $4.902 \pm 0.154$ & $5.005 \pm 0.111$ \\
\hline $1.0 \mathrm{mg}$ & & $5.065 \pm 0.331$ & $3.743 \pm 0.274$ & $4.317 \pm 0.246$ \\
\hline $3.0 \mathrm{mg}$ & $\downarrow$ & $3.965 \pm 0.276$ & $3.222 \pm 0.298$ & $3.392 \pm 0.191$ \\
\hline
\end{tabular}

The mean startle magnitude (In-transformed in arbitrary units +SEM) obtained in the first six, the last six pulse-alone trials, and across the 12 pulse-alone trials (presented in the middle of the session, intermixed with other trial types) of Experiment I are summarized. The arrows indicate whether the treatment led to a significant enhancement $(\uparrow)$ or reduction $(\downarrow)$ in average startle reactivity relative to saline-treated controls $(p<0.05)$. 
a

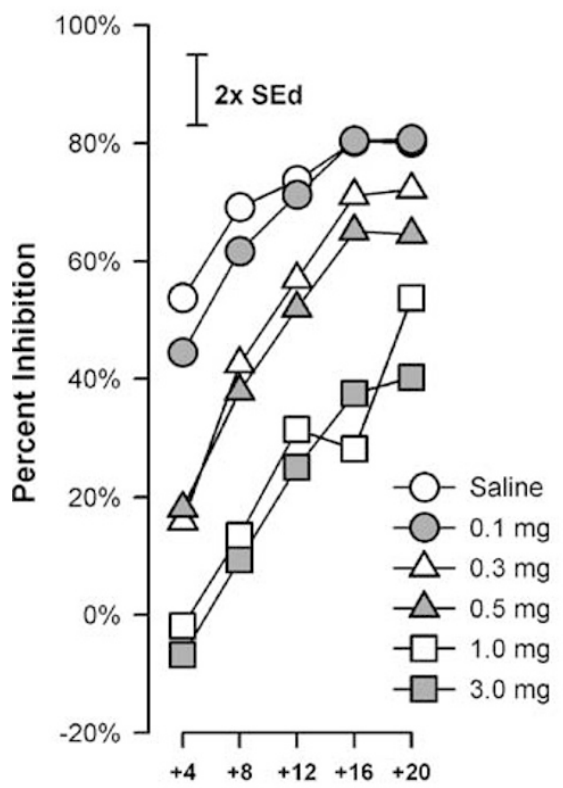

Prepulse intensity ( $\mathrm{dB}_{\mathrm{A}}$ above background) b

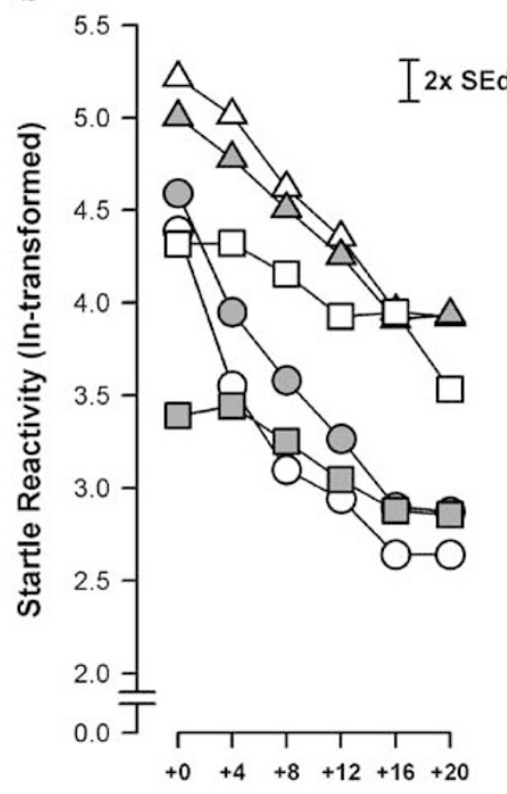

Prepulse intensity ( $\mathrm{dB}_{\mathrm{A}}$ above background)
C

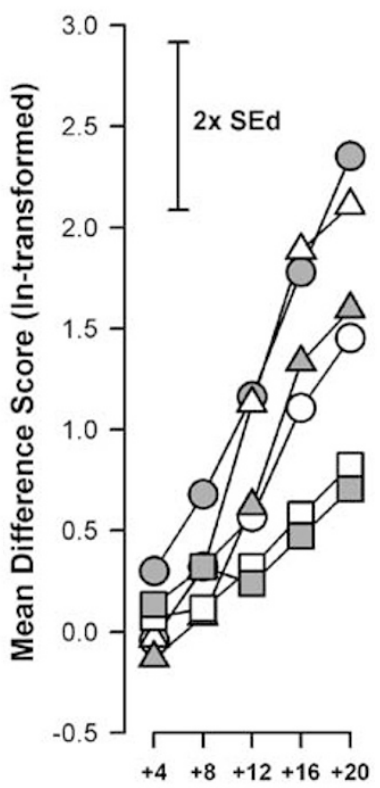

Prepulse intensity ( $\mathrm{dB}_{\mathrm{A}}$ above background)

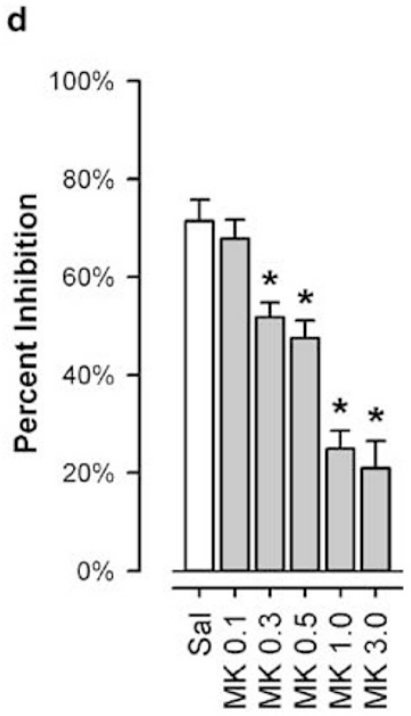

Treatment e

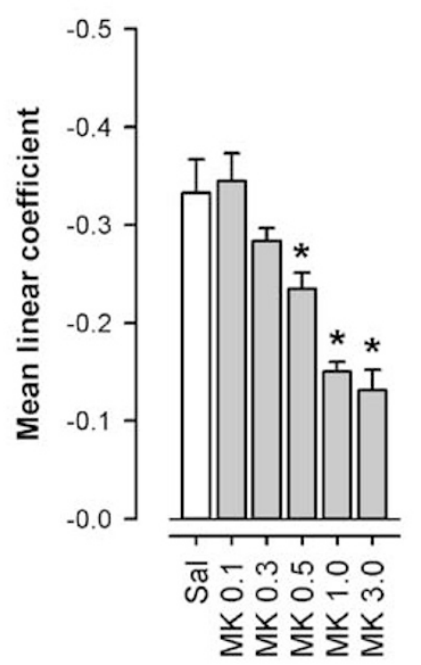

Treatment f

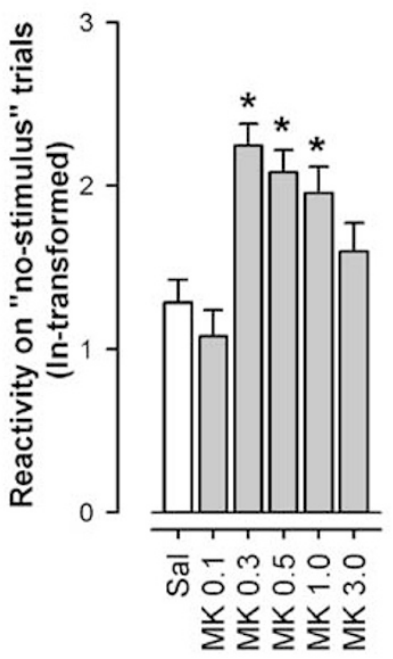

Treatment g

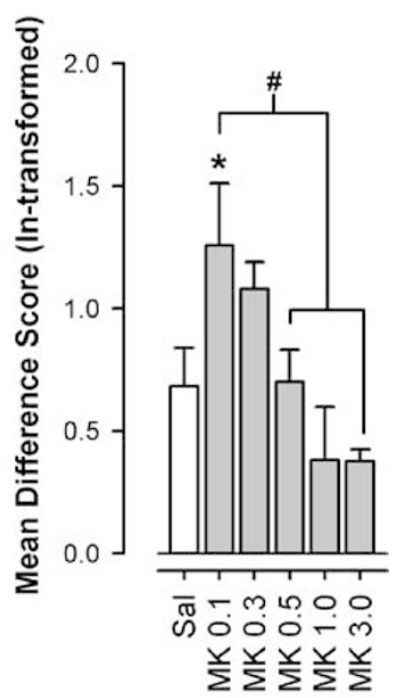

Treatment

Figure I Effects of systemic dizocilpine on PPI, prepulse-elicited reactivity, and reactivity on no-stimulus trials. (a) Mean percent inhibition (\%PPI) is expressed as a function of prepulse intensity ( $\mathrm{dB}$ above background) across treatment conditions, with the average magnitude of \%PPI across the five levels of prepulse intensities illustrated in (d). (b) Mean reactivity (In-transformed) obtained on pulse-alone and prepulse-plus-pulse trials as a function of prepulse intensity (dB above background; ' + 0' refers to pulse-alone trials), across treatment conditions, with the mean linear coefficient derived for each treatment condition depicted in (e). (c) Prepulse-elicited reactivity is represented as mean difference score relative to 'no-stimulus' trials (In-transformed) as a function of prepulse intensity ( $\mathrm{dB}$ above background) for each treatment condition, with the mean difference score in each treatment condition depicted in ( $\mathrm{g}$ ). ( $\mathrm{f}$ ) The mean reactivity score (In-transformed) derived from 'no-stimulus' trials. (a)-(c): The symbols $\bigcirc, \mathbf{O}, \triangle, \mathbf{\Delta}, \square, \mathbf{\square}$ refer to saline, $0.1,0.3,0.5,1,3 \mathrm{mg} / \mathrm{kg}$ of dizocilpine, respectively. Error bars refer to twice the standard errors of difference $(2 \times \mathrm{SEd})$ derived from the error terms in the appropriate ANOVA table. (d)-(g): Error bars refer to the standard errors of the mean. * refers to a significant difference relative to saline controls. \# refers to significant difference between $0.1 \mathrm{mg} / \mathrm{kg}$ MK-80I against $0.5 \sim 3.0 \mathrm{mg} / \mathrm{kg}$ MK-80I. All post-hoc comparisons were based on Fisher's LSD, significant levels set at $p<0.05$.

Reactivity scores: Given that there was a significant main effect of treatment in startle reactivity on pulse-alone trials (see above), it was deemed necessary to conduct additional analysis on the reactivity scores including the scores obtained on pulse-alone trials (Swerdlow et al, 2000). To improve the data distribution and variance homogeneity, the reactivity scores were first logarithmically transformed, before being submitted to a $6 \times 6$ (treatment $\times$ prepulse 
intensities) split-plot ANOVA (as explained in the Methods). As shown in Figure 1b, the presence of a prepulse prior to the pulse stimulus reduced startle reactivity to the latter, and this effect was stronger when the corresponding prepulse stimulus was more intense (prepulse intensities: $\mathrm{F}(5,215)=215.91, p<0.001)$. As a function of prepulse intensities, reactivity to the pulse stimulus (in prepulseplus-pulse trials) showed a clear downward trend, and this constitutes the PPI effect. The magnitude of the PPI effect was, however, not uniformly expressed in all treatment groups, as evident by the significant treatment $\times$ prepulse intensities interaction $(\mathrm{F}(25,215)=7.18, p<0.001)$ with a characteristic linear trend $(\mathrm{F}(5,43)=14.18, p<0.001)$. As depicted in Figure 1e, the mean linear coefficient derived for each treatment condition conformed closely to the impression obtained in the percent inhibition analysis, as summarized in Figure 1d. Thus, both analyses (percent inhibition and reactivity scores) indicated a clear dosedependent PPI disruption by dizocilpine.

Finally, an ANCOVA of percent PPI with startle reactivity (ln-transformed) as the covariate was carried out to address the possibility that dizocilpine's effect on PPI could be explained by the drug's effect on startle reactivity. It showed that the main effect of treatment remained highly significant $(\mathrm{F}(5,42)=22.07, p<0.001)$, with the covariate not attaining statistical significance $(\mathrm{F}<1.0, n s)$.

Reactivity on 'no-stimulus' trials. Separate analysis of nostimulus trials allowed an evaluation of the effect of the drug on the spontaneous whole-body motion. A one-way ANOVA on the ln-transformed mean scores obtained on the no-stimulus trials yielded a significant effect of treatment $(F(5,43)=8.13, p<0.001)$ (Figure 1f). Pair-wise Fisher's LSD comparisons revealed that dizocilpine at doses $0.3,0.5$, and $1.0 \mathrm{mg} / \mathrm{kg}$ significantly enhanced reactivity recorded on no-stimulus trials (all p's $<0.01$ ). The lowest and highest doses examined, however, exerted no effect on this measure.

Prepulse-elicited reactivity. Prepulse-elicited reactivity was captured by the use of a difference score (as deviation from 'no-stimulus' trials). As expected, prepulse-elicited reactivity increased as a function of prepulse intensities, but the responsiveness to this effect differed among treatment groups. It can be readily discerned in Figures $1 \mathrm{c}$ and $\mathrm{g}$ that the effects of dizocilpine upon prepulse-elicited reactivity were bi-directional relative to saline control. Lower doses of dizocilpine enhanced prepulse-elicited reactivity, while higher doses clearly lacked such an effect, and even exhibited a non-significant trend toward prepulse-elicited reactivity reduction. The divergent effects at the lower and higher doses were more apparent when the prepulse intensity reached $+12 \mathrm{~dB}$ and beyond (see Figure $1 \mathrm{c}$ ).

These interpretations were supported by the main effect of prepulse intensities $(\mathrm{F}(4,172)=98.68, p<0.001)$, of treatment $(\mathrm{F}(5,43)=4.42, p<0.01)$, and their interaction $(\mathrm{F}(20,172)=3.90, p<0.001)$. Post hoc analyses of the main treatment effect showed that the dose of $0.1 \mathrm{mg} / \mathrm{kg}$ was associated with a significant overall enhancement of prepulse-elicited reactivity relative to control, and relative to the three highest doses of dizocilpine examined here (all $p$ 's $<0.05$ ) (see Figure 1g).

To examine if the effects on prepulse-elicited reactivity could be accounted for by the drug's concomitant effect observed on pulse-alone trials, we conducted an additional analysis of covariance of the prepulse-elicited reactivity data set using the mean reactivity scores (ln-transformed) on the middle 12 pulse-alone trials, which were presented intermixed with other trial types and were used to calculate $\%$ PPI, as covariate. The ANCOVA yielded a significant effect of covariate $(\mathrm{F}(1,42)=9.01, p<0.01)$, and yet the main effect of treatment remained significant $(\mathrm{F}(5,42)=3.12, p<0.05)$.

Similarly, an ANCOVA was performed with mean reactivity (ln-transformed) obtained on no-stimulus trials as covariate. This yielded a significant effect of covariate $(\mathrm{F}(1,42)=15.52, p<0.001)$. The main effect of treatment $\mathrm{F}(5,42)=7.00, p<0.001)$ as well as the interaction between treatment and prepulse intensities $(\mathrm{F}(20,168)=3.88$, $p<0.001)$ remained highly significant.

\section{Experiment 2: Effects of PCP on PPI and Prepulse- Elicited Reactivity}

Startle habituation. Startle habituation was evident by a significant overall reduction of startle reactivity in the last six pulse-alone trials in comparison to the first six pulsealone trials $(\mathrm{F}(1,42)=6.83, p<0.05)$. Inspection of individual groups indicated a trend towards startle habituation in all treatment groups except PCP/15 mg (Table 2). The interaction between treatment and blocks, however, was not significant $(\mathrm{F}(5,42)=1.36, p=0.261)$, supporting the conclusion that startle habituation did not differ significantly among groups. PCP had a tendency to enhance startle reactivity as such, but this effect was not statistically significant $(\mathrm{F}(5,42)=2.21, p=0.071)$.

Startle reactivity. Analysis of the 12 pulse-alone trials presented in the middle of the session, intermixed with other trial-types, also suggested a similar drug effect (see

Table 2 Effects of Phencyclidine (PCP) on Startle Habituation and Startle Reactivity

Mean startle reactivity (In-transformed)

\begin{tabular}{lccc}
\cline { 2 - 4 } & $\begin{array}{c}\text { First six } \\
\text { pulse-alone } \\
\text { (consecutive) } \\
\text { trials }\end{array}$ & $\begin{array}{c}\text { Last six } \\
\text { pulse-alone } \\
\text { (consecutive) } \\
\text { trials }\end{array}$ & $\begin{array}{c}\text { Middle I2 } \\
\text { pulse-alone } \\
\text { (distributed) } \\
\text { trials }\end{array}$ \\
\hline Saline & $4.148 \pm 0.35 \mid$ & $4.021 \pm 0.170$ & $4.313 \pm 0.215$ \\
$2.5 \mathrm{mg}$ & $4.914 \pm 0.234$ & $4.557 \pm 0.119$ & $4.946 \pm 0.27 \mid$ \\
$5.0 \mathrm{mg}$ & $4.664 \pm 0.228$ & $4.584 \pm 0.149$ & $4.869 \pm 0.157$ \\
$7.5 \mathrm{mg}$ & $5.008 \pm 0.227$ & $4.348 \pm 0.112$ & $4.613 \pm 0.111$ \\
$10.0 \mathrm{mg}$ & $5.187 \pm 0.231$ & $4.590 \pm 0.150$ & $5.003 \pm 0.098$ \\
$15.0 \mathrm{mg}$ & $4.363 \pm 0.377$ & $4.471 \pm 0.180$ & $4.326 \pm 0.254$ \\
\hline
\end{tabular}

The mean startle magnitude (In-transformed in arbitrary units +SEM) obtained in the first six, the last six pulse-alone trials, and across the 12 pulse-alone trials (presented in the middle of the session, intermixed with other trial types) of Experiment 2 are summarized. 
Table 2), and again was close to but failed to reach statistical significance $(\mathrm{F}(5,42)=2.39, p=0.054)$.

\section{Prepulse inhibition.}

\%PPI: PCP also disrupted PPI in a dose-dependent manner as confirmed by the analysis of percent PPI, and the analysis of reactivity scores. Analysis of percent PPI yielded a main effect of prepulse intensities $(F(4,168)=121.05$, $p<0.001)$, of treatment $(\mathrm{F}(5,42)=17.33, p<0.001)$, and of their interaction $(\mathrm{F}(20,168)=2.27, p<0.01)$ (Figures 2a and d). PCP was effective in attenuating PPI at all doses examined, as confirmed by post hoc comparisons against saline controls (all $p<0.05)$.

Reactivity scores: A $6 \times 6$ (treatment $\times$ prepulse intensities) ANOVA of the reactivity scores on pulse-alone and prepulse-plus-pulse trials yielded a significant interaction $(\mathrm{F}(25,210)=6.12, p<0.001)$. The presence of this interaction suggests that the effectiveness of the prepulse in attenuating startle reaction to the subsequent pulse stimulus (ie the PPI effect) differed among groups (Figure 2b). Again, this interaction was highly significant in the linear trend $(\mathrm{F}(5,42)=10.94, p<0.001)$. As illustrated in Figure 2e, the linear components derived from the reactivity score data provided a striking resemblance to the impression obtained in the percent PPI analysis, as summarized in Figure $2 \mathrm{~d}$.

ANCOVA of percent PPI with startle reactivity as the covariate further confirmed that the disruption of PPI could not be accounted for by the drug's (nonsignificant) effect on startle reactivity. The main effect of treatment remained highly significant in the ANCOVA $(F(5,41)=16.78$, $p<0.001$ ), with the covariate failing to attain significance $(\mathrm{F}<1.0, n s)$.

Reactivity on 'no-stimulus' trials. Separate analysis of nostimulus trials allowed an evaluation of the effect of the drug on the spontaneous whole-body motion. A one-way ANOVA on the ln-transformed mean scores obtained on the no-stimulus trials yielded a significant effect of treatment $(F(5,42)=8.13, p<0.001)$ (see Figure 2f). Pairwise Fisher's LSD comparison revealed that PCP at doses $7.5,10$, and $15 \mathrm{mg} / \mathrm{kg}$ significantly enhanced reactivity recorded on no-stimulus trials (all $p<0.01$ ), whereas the lower two doses had no effect.

Prepulse-elicited reactivity. As illustrated in Figures $2 \mathrm{c}$ and $\mathrm{g}$, the shape of the dose-dependent effects of PCP on prepulse-elicited reactivity resembled that of dizocilpine. Across the five doses examined, response magnitude towards the prepulse stimulus decreased as the dosage increased (see Figure 2g). Analysis of the prepulse-elicited reactivity data yielded a main effect of prepulse intensities $(\mathrm{F}(4,168)=112.52, p<0.001)$. This was further accompanied by a significant interaction with treatment $(\mathrm{F}(20,168)=3.19, p<0.001)$, suggesting that the sensitivity to prepulse-elicited reaction was not uniform across treatment groups (see Figure 2c). At the lowest dose $(2.5 \mathrm{mg} / \mathrm{kg})$, PCP marginally enhanced prepulse-elicited reactivity. Although this effect was nonsignificant, reactivity at this dose was significantly higher than the three highest doses $(7.5-15 \mathrm{mg} / \mathrm{kg})($ all $p$ 's $<0.05)$. At successively higher doses, there was a clear trend towards reduced prepulseelicited reactivity, and maximal reduction was observed at $10 \mathrm{mg} / \mathrm{kg}$ when it reached a level significantly below that of the control $(p<0.05)$ (see Figure $2 \mathrm{~g}$ ).

Further analysis of covariance (ANCOVA) suggested that the observed effect of PCP on prepulse-elicited reactivity was not significantly related to, nor explained by, the drug's effects on startle reactivity in pulse-alone trials. In an ANCOVA with mean pulse-alone reactivity (ln-transformed) over the middle 12 pulse-alone trials as covariate, the treatment $\times$ prepulse intensities interaction term was found to remain highly significant $(F(20,164)=3.10$, $p<0.001$ ), which was further accompanied by a significant main effect of treatment $(\mathrm{F}(5,41)=2.61, p<0.05)$. Unlike the equivalent analysis of Experiment 1, the covariate failed to achieve statistical significance $(\mathrm{F}(1,41)=3.04, p=0.089)$.

Similarly, an ANCOVA was conducted with the mean reactivity (ln-transformed) obtained on no-stimulus trials as covariate in order to gauge if the observed effect of PCP on prepulse-elicited reactivity could be solely attributed to the drug's effects on spontaneous reactivity obtained on nostimulus trials. This yielded a significant covariate effect $(\mathrm{F}(1,41)=36.28, p<0.001)$; yet the interaction between treatment and prepulse intensities revealed by the original ANOVA remained significant $(\mathrm{F}(20,164)=2.03, p<0.01)$.

\section{Correlative Analyses (Experiments 1 and 2)}

Three separate correlative analyses were carried out to investigate the relationship between the magnitude of PPI and the magnitude of prepulse-elicited reactivity. One analysis included all subjects in Experiment 1 (Figure 3a), and another all subjects in Experiment 2 (Figure 3b). The third analysis included only saline-treated controls taken from both experiments (Figure 3c). The two variables correlated significantly with each other in all three analyses (Experiment 1: $r=+0.442, \mathrm{df}=47, p<0.01$; Experiment 2: $r=+0.361, \quad \mathrm{df}=46, \quad p<0.05 ; \quad$ saline-treated subjects: $r=+0.533, \mathrm{df}=14, p<0.05)$, showing that higher levels of prepulse-elicited reactivity were associated with stronger prepulse inhibition.

In all three cases, the correlation remained highly significant even when the two variables' covariance with pulse-alone reactivity was controlled in a partial correlative analysis (Experiment 1: $r=+0.362, \mathrm{df}=46, \quad p<0.02$; Experiment 2: $r=+0.398, \mathrm{df}=45, p<0.01$; saline-treated subjects: $r=+0.691, \mathrm{df}=13, p<0.005)$.

\section{DISCUSSION}

As expected, dizocilpine and PCP dose-dependently disrupted PPI in mice, and the dose dependency profile reported here is in general agreement with previous reports based on the C57BL6 strain (eg Varty et al, 2001). The novel findings here concern the two drugs' concomitant effects on prepulse-elicited reactivity. These appeared to be readily distinguishable from that of apomorphine, which enhances prepulse-elicited reactivity at a dose that disrupts PPI (Yee et al, 2004). In contrast, at doses that were highly effective in disrupting PPI here, both dizocilpine and PCP tended to reduce prepulse-elicited reactivity. Although this effect only 


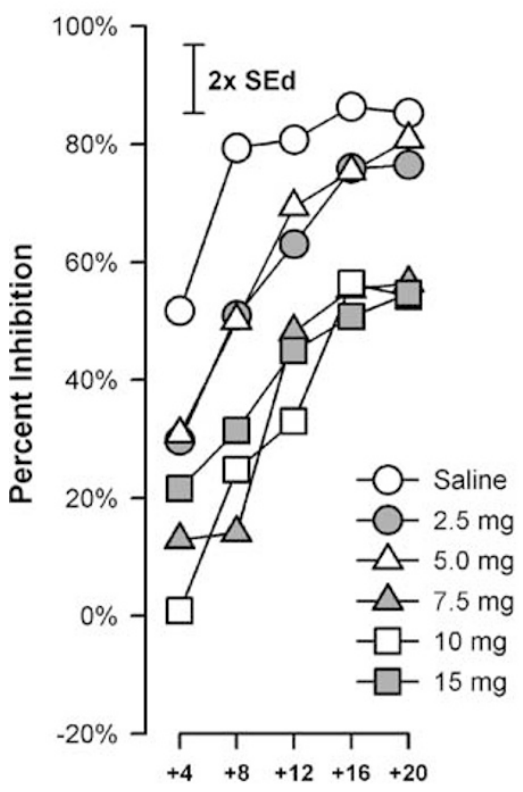

Prepulse intensity ( $\mathrm{dB}_{\mathrm{A}}$ above background) b

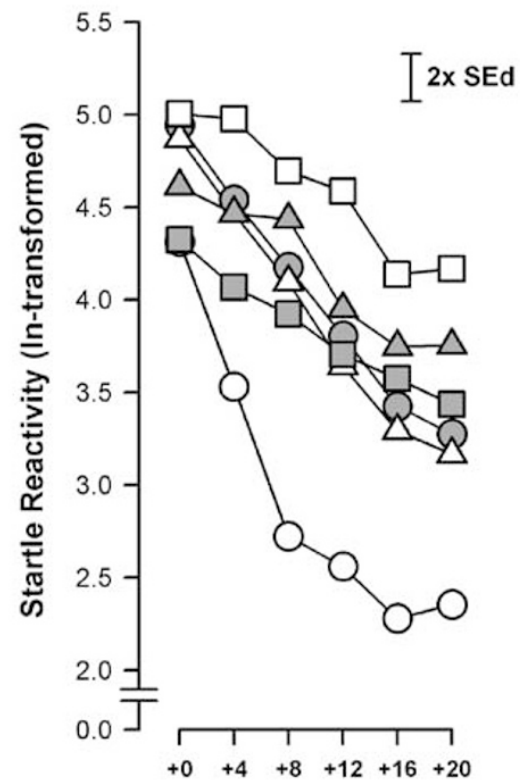

Prepulse intensity ( $\mathrm{dB}_{\mathrm{A}}$ above background)

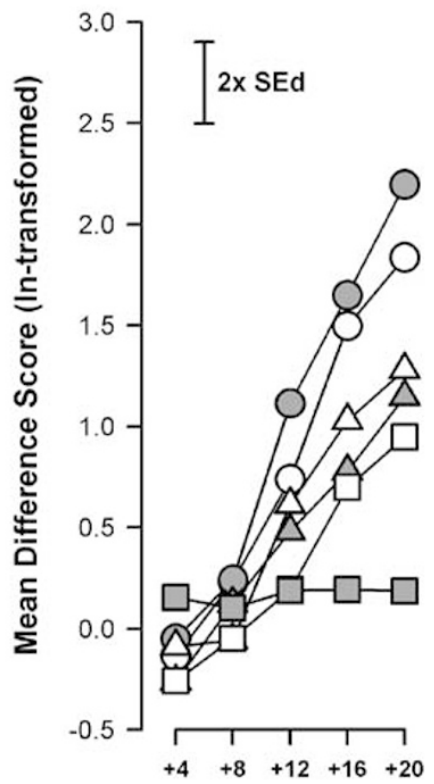

Prepulse intensity ( $\mathrm{dB}_{\mathrm{A}}$ above background)

\section{g}

d

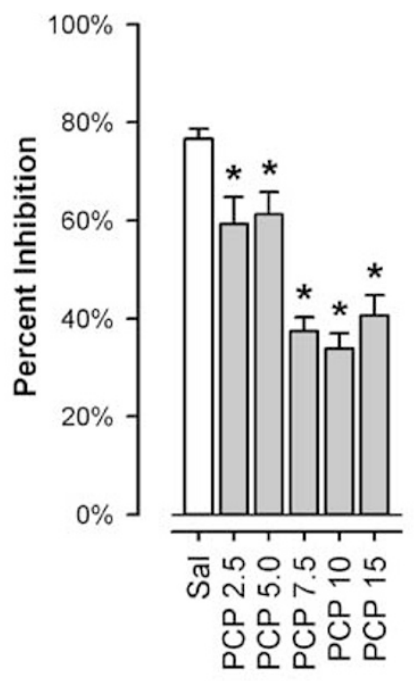

Treatment e

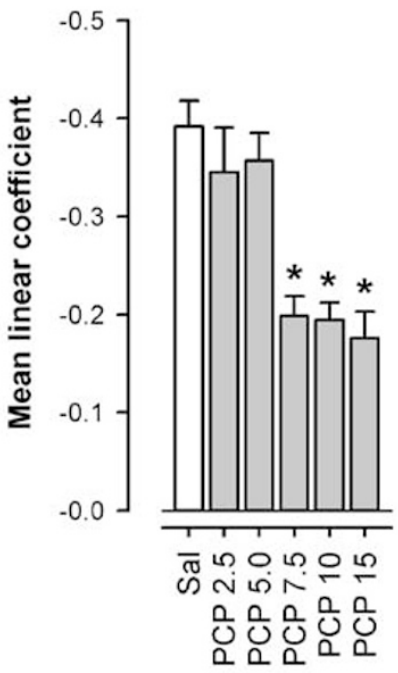

Treatment

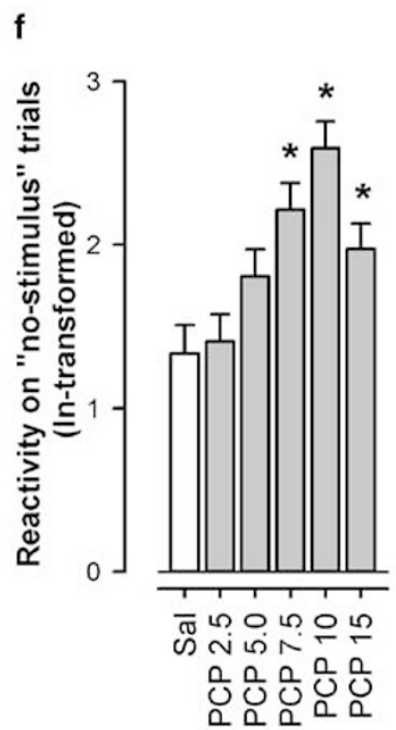

Treatment

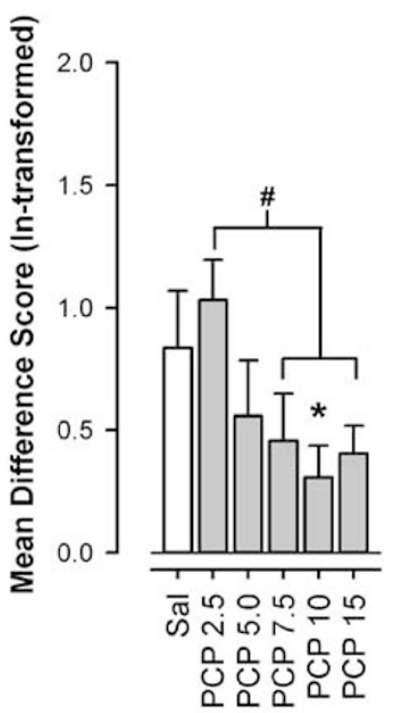

Treatment

Figure 2 Effects of systemic PCP on PPI, prepulse-elicited reactivity, and reactivity on no-stimulus trials. (a) Mean percent inhibition (\%PPI) is expressed as a function of prepulse intensity ( $\mathrm{dB}$ above background) across treatment conditions, with the average magnitude of \%PPI across the five levels of prepulse intensities illustrated in (d). (b) Mean reactivity (In-transformed) obtained on pulse-alone and prepulse-plus-pulse trials as a function of prepulse intensity (dB above background; ' + 0 ' refers to pulse-alone trials), across treatment conditions, with the mean linear coefficient derived for each treatment condition depicted in (e). (c) Prepulse-elicited reactivity is represented as mean difference score relative to 'no-stimulus' trials (In-transformed) as a function of prepulse intensity ( $\mathrm{dB}$ above background) for each treatment condition, with the mean difference score in each treatment condition depicted in ( $\mathrm{g}$ ). ( $\mathrm{f}$ ) the mean reactivity score (In-transformed) derived from 'no-stimulus' trials. (a)-(c): The symbols $\bigcirc, \boldsymbol{Q}, \triangle, \boldsymbol{\Delta}, \square, \boldsymbol{\square}$ refer to saline, 2.5, 5.0, 7.5, I0 and I5 mg/ $\mathrm{kg}$ of PCP, respectively. Error bars refers to twice the standard errors of difference $(2 \times \mathrm{SEd})$ derived from the error terms in the appropriate ANOVA table. (d)-(g): Error bars refer to the standard errors of the mean. * refers to a significant difference relative to saline controls. \# refers to significant difference between $2.5 \mathrm{mg} / \mathrm{kg}$ PCP against $7.5 \sim 15 \mathrm{mg} / \mathrm{kg}$ PCP. All post-hoc comparisons were based on Fisher's LSD, significant levels set at $p<0.05$. 
a

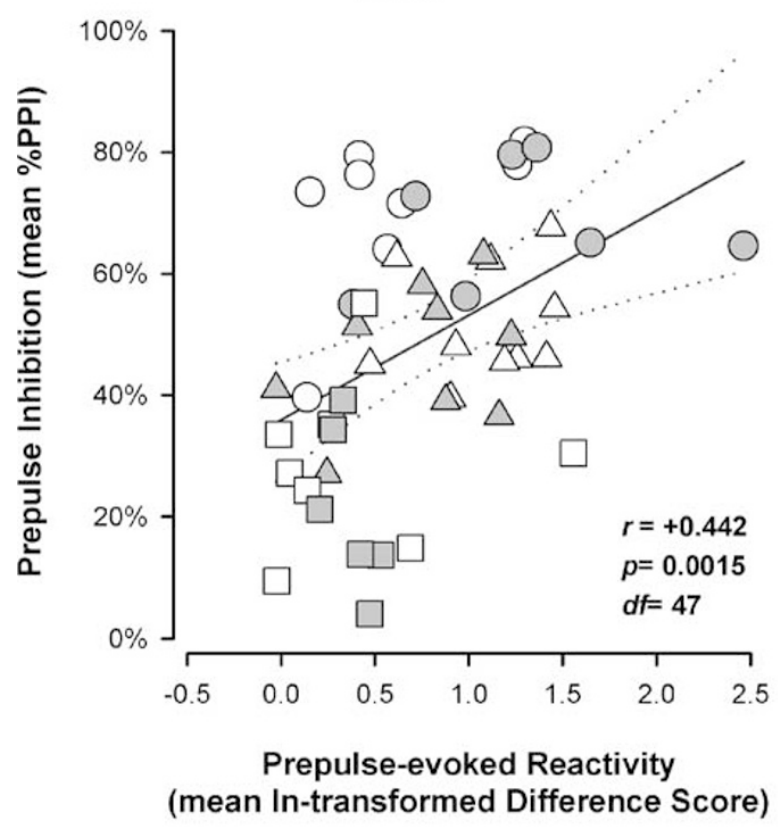

b $\quad P C P$

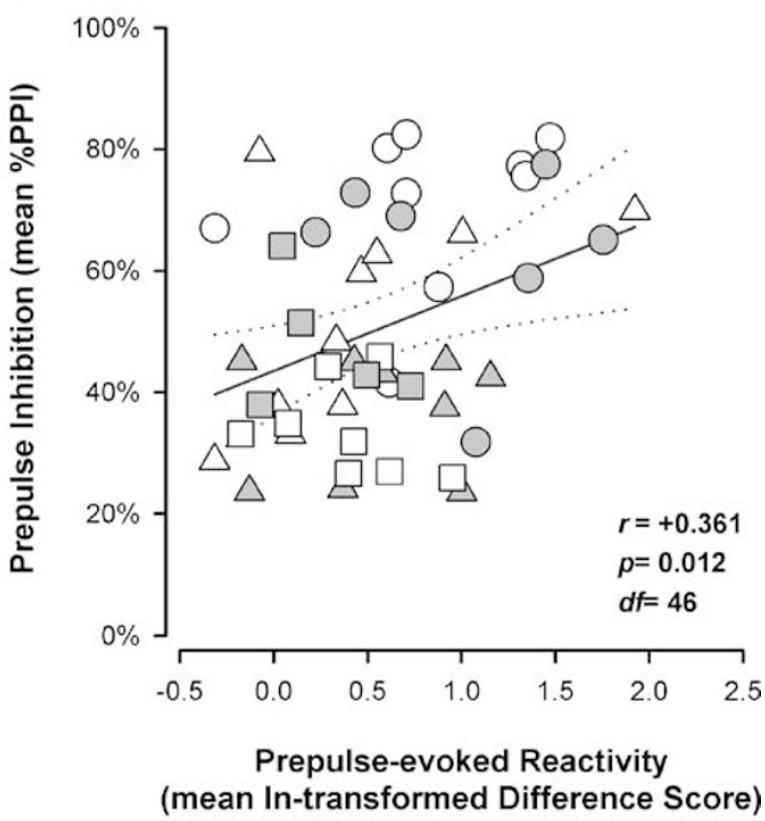

C

Saline

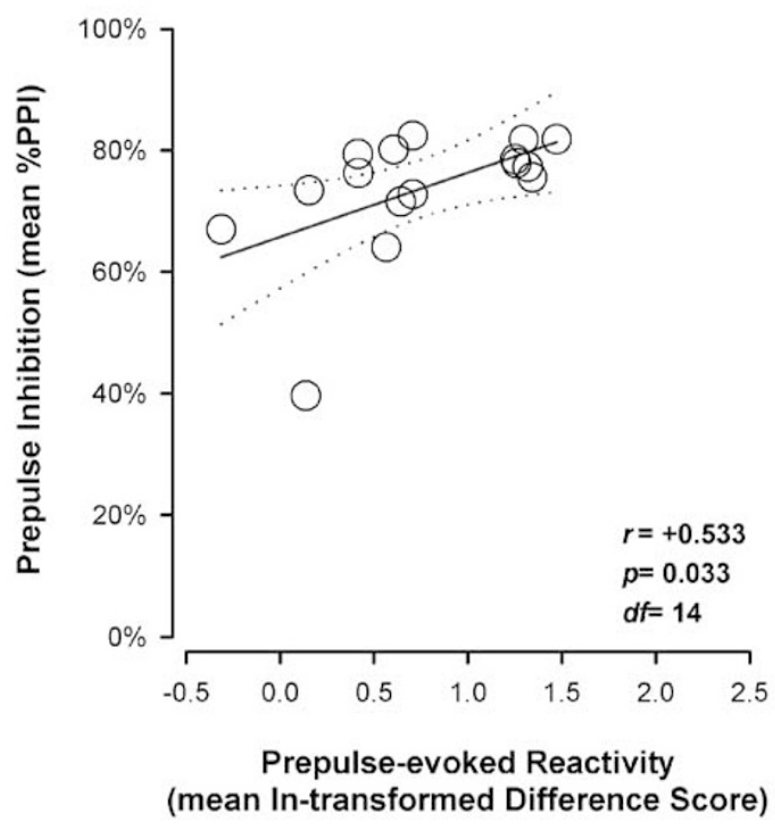

Figure 3 Results of the correlative analysis. Scatter plots of average percent prepulse inhibition against average prepulse-evoked reactivity (mean Intransformed difference score) of the data derived from all subjects in Experiment I with dizocilpine (a) and of the data derived from all subjects in Experiment 2 with PCP (b). The saline-treated subject taken from the two experiments were combined in a separate plot (c). The symbols in (a): $\bigcirc$, $\triangle, \triangle$, $\boldsymbol{\Lambda}, \square, \boldsymbol{\square}$ refer to saline, $0.1,0.3,0.5,1,3 \mathrm{mg} / \mathrm{kg}$ of dizocilpine, respectively. The symbols in (b): $\bigcirc, \boldsymbol{Q}, \triangle, \boldsymbol{\Delta}, \square, \boldsymbol{\square}$ refer to saline, 2.5, 5.0, 7.5, I0 and $15 \mathrm{mg} / \mathrm{kg}$ of PCP, respectively.

attained statistical significance in Experiment 2 with PCP, a trend in the same direction seemed evident in Experiment 1.

This attenuating effect clearly contrasted with the drugs' effects at low doses, when reactivity to the prepulse was surprisingly enhanced. Again, the enhancing effect was significant with one drug (dizocilpine) but not the other (PCP). Notably, when dizocilpine (at $0.1 \mathrm{mg} / \mathrm{kg}$ ) was most effective in enhancing prepulse-elicited reactivity (see Figure 1g), it was clearly without an effect on the expression of PPI (see Figure 1d), which contrasted with the enhancing effect seen following apomorphine treatment as PPI was disrupted at the same time (Yee et al, 2004). Across the five doses examined, both drugs exhibited a clear dosedependent reduction of prepulse-elicited reactivity. In 
comparison, the control level of prepulse-elicited reactivity fell in between the prepulse-elicited reactivity observed with the lowest and the highest doses of the drug. Hence, the effect of dizocilpine and PCP on prepulse-elicited reactivity appeared bi-directional, with prepulse-elicited reactivity enhanced at low doses but attenuated at higher doses.

\section{Comparison with the Effects of Apomorphine}

Is it possible that a dose of dizocilpine and of PCP exists such that a pattern of results conforming to that following apomorphine treatment can be convincingly demonstrated? Considering the dose-response curves of both compounds on prepulse-elicited reactivity and prepulse inhibition, it seems highly unlikely that doses that maximally enhance prepulse-elicited reactivity would be associated with the most severe PPI disruption. On the other hand, the converse is more likely, because PCP significantly attenuated both prepulse-elicited reactivity and PPI at higher doses. Although these dual-attenuating effects were not as clearly seen with dizocilpine at higher doses, a closer match would be expected if doses beyond $3 \mathrm{mg} / \mathrm{kg}$ had been included in the present study. A related question could be raised as to whether apomorphine may also exhibit a bi-directional effect on prepulse-elicited reactivity. It should be pointed out that the enhancement of prepulse-elicited reactivity following apomorphine treatment was demonstrated at one dose $(2 \mathrm{mg} / \mathrm{kg}$, s.c.) (Yee et al, 2004). Our unpublished data obtained with a lower (non-PPI-disrupting) dose did not yield an opposite effect. It therefore remains to be tested if higher doses might reduce response to the prepulse stimuli. However, even if this is the case, apomorphine should significantly disrupt PPI at doses beyond $2 \mathrm{mg} / \mathrm{kg}$, and thus would still be different from the pattern of results obtained here with the lower doses of dizocilpine and PCP, when they were minimally effective in disrupting PPI.

Dizocilpine, PCP, and apomorphine all reliably disrupt PPI in rodents, but recent evidence suggests that the neuropharmacology of PPI clearly differs between rats and mice (eg Ralph-Williams et al, 2003). Hence, the discussion of the present novel findings regarding prepulse-elicited reactivity should be restricted to the mouse until similar results are also demonstrated in rats. This restriction, however, does not undermine the significance of the present findings, because of the increasing use of transgenic mice. In this respect, one should further qualify that the present data were obtained in one mouse strain alone, albeit a very commonly employed strain. Thus, the generality of our findings across strains also awaits future evaluation.

Based on our present and previous studies in C57BL6 mice, the data indicate that dizocilpine and PCP at doses associated with the clearest PPI disruption behave differently from apomorphine in terms of their concomitant effects on prepulse-elicited reaction. One extended interpretation is that NMDA antagonists tend to exert an effect on prepulse-elicited reactivity opposite to that of apomorphine, namely, an attenuation of prepulse-elicited reaction. Additional experiments are warranted to further qualify the latter, more generalized claim. Nonetheless, the present data suggest a possible means to distinguish behaviorally between PPI disruptions seen following treatment of pharmacologically distinct classes of drugs. Dizocilpine and PCP resemble each other in their dose-response relationship with respect to prepulse-elicited reactivity as well as PPI, and this leads one to suspect that the pattern observed here might be primarily attributable to their common pharmacological effect, namely, NMDA-receptor antagonism.

\section{Relationship between Prepulse-Elicited Reactivity and PPI}

The present study further replicated the previous observation of a positive correlation between prepulse-elicited reactivity and PPI in C57BL6 mice (Yee et al, 2004). This was demonstrated in both experiments here, and when the saline-treated control animals from the two experiments were pooled for a single correlative analysis. Furthermore, the correlation observed was demonstrably not mediated by covariance with startle reactivity. This lends support to the notion that prepulse reaction and/or processing is related to the expression of PPI as predicted by Graham's theoretical account of PPI (Graham, 1975, 1980, 1992; also see Dahmen and Corr, 2004).

The possibility that a manipulation may disrupt PPI via an effect on prepulse detectability has been suggested by Davis et al (1990). This hypothesis was originally developed to account specifically for apomorphine-induced PPI disruption, but it seems to fit the present results at high doses, especially PCP. Yet, it does not necessarily follow that, when a manipulation leads to concomitant suppression of prepulse-elicited reactivity and PPI, the two effects are causally related. Although causality cannot be evaluated statistically, we employed an ANCOVA to test whether the PPI disrupting effect induced by PCP at $10 \mathrm{mg} / \mathrm{kg}$ could be solely accounted for by the drug's clear effect on prepulseelicited reactivity at this dose. An ANCOVA comparing $\%$ PPI between vehicle-treated control subjects and animals treated with PCP at $10 \mathrm{mg} / \mathrm{kg}$, with prepulse-elicited reactivity as covariate, indicated that the main effect of PPI disruption by PCP $10 \mathrm{mg} / \mathrm{kg}$ remained highly significant $(F(1,12)=93.76, p<0.001)$. This undermines the interpretation that PPI disruption can be explained solely by changes in prepulse-elicited reactivity induced by the same drug, despite the fact that the two effects are clearly associated statistically and on theoretical grounds (eg Graham, 1975, 1980, 1992). Hence, even if reduced prepulse detectability had contributed to the observed PPI attenuation, other processes would have to be involved. A similar conclusion can be drawn when one considers the modest effect size of the correlation between prepulse-elicited reactivity and \%PPI, which accounted for $10-20 \%$ of the variance. This suggests that, while detection and/or processing of the prepulse plays a role, its influence may not be overriding.

We suggest that additional factors can directly influence the gating mechanism without acting via prepulse detection. A disruption of gating mechanism which underlies the expression of PPI either by acting as a perceptual filter (Braff et al, 1992) or by means of an active inhibition of pulse processing (Graham, 1975) will be expected to attenuate PPI. Thus, while prepulse detection is, by definition, the trigger of the gating mechanism underlying PPI, the gating process itself, once initiated, is also under 


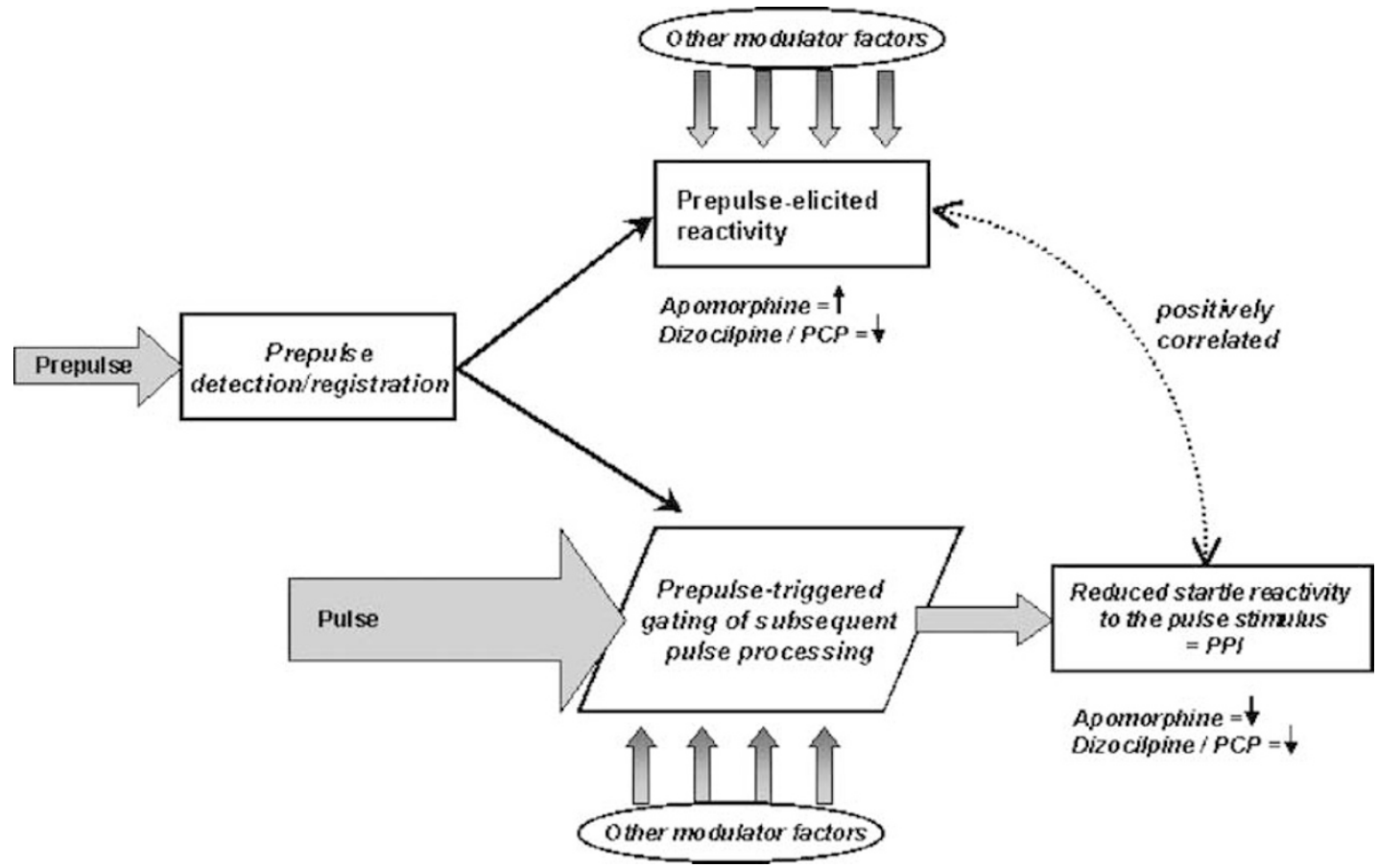

Figure 4 A diagrammatic representation of the interplay between prepulse processing and prepulse inhibition. Detection of the prepulse stimulus is considered to initiate two largely independent processes. One leads to the emergence of prepulse-elicited reaction, which is considered as an indirect measure of prepulse processing. Another triggers the gating or inhibitory process which leads to the reduced reaction to the subsequent pulse stimulus. These two processes induced by the presentation of the prepulse stimulus are assumed to underlie the positive correlation observed between prepulseelicited reactivity and the magnitude of prepulse inhibition. Additional modulating factors are postulated to be in a position to affect differentially the efficacy of the gating mechanisms and the strength of prepulse-elicited response.

the modulation of factors largely unrelated to the detection/ processing of the prepulse (see Figure 4). It is therefore feasible that a manipulation impairs sensorimotor gating via an influence on such factors, regardless of whether it exerts any influence on the detection of prepulse or the initiation and execution of prepulse-elicited reactivity.

As represented in Figure 4, prepulse detection or registration is responsible for the prepulse-elicited reactivity we observed, and is attributed to a role in triggering the gating of subsequent pulse processing, which gives rise to PPI. Our data derived from drug-induced PPI disruption in mice support the conclusion that the two processes are largely independent, in spite of the positive correlation between prepulse-elicited reactivity and PPI. The most compelling evidence comes from the study with apomorphine, which yielded a pattern of results which we even referred to as paradoxical. In that study, the concomitant effects of apomorphine on prepulse-elicited reactivity (an elevation) and on PPI (an attenuation) were demonstrated against the background of a positive correlation between the two variables within the apomorphine group (Yee et al, 2004). Therefore, PPI disruption can indeed be associated with any possible changes in prepulse-elicited reactivity when the effects of apomorphine, dizocilpine, and PCP are all considered together (also see Yee, 2000).

\section{Application and Implications of Prepulse-Elicited Reactivity Analysis in PPI Studies}

Does any alteration in prepulse-elicited reactivity merely represent an epiphenomenon not worth considering ser- iously in the neuropsychopharmacological and clinical studies of PPI? Given that prepulse processing is an integral part of the PPI phenomenon, we believe that direct measures of the processing of prepulse stimulus are of value in the PPI studies and should be encouraged. Our current measures were relatively crude and relied on the same mechanistic device designed first and foremost to measure whole-body startle reaction. We have therefore refrained from interpreting our prepulse-elicited measures beyond that of an index of prepulse detection or processing. Additional characterization based on EEG and behavioral signs of orienting (eg pinna movement), as suggested by an anonymous reviewer, should help in identifying the physiological nature of the current prepulse-elicited responses.

Given these caveats, the analysis of prepulse-elicited reactivity has nonetheless provided us a means to distinguish between two classes of PPI-disrupting drugs. This behavioral distinction supports the suggestion that the neural substrate underlying PPI disruption induced by dopamine agonists and by NMDA antagonists can be differentiated (eg Kretschmer and Koch, 1998). Although their respective concomitant effect on prepulse-elicited startle could not satisfactorily explain the drugs' disruptive effect on PPI, they could be useful in the understanding of their psychopharmacology in general. Previously, we postulated that the dual effects of apomorphine upon PPI and prepulse-elicited reactivity might be indicative of enhanced distractibility, and the treated mice were described as reacting to stimuli (prepulse or pulse following a prepulse) that normal subjects tended to process less (Yee 
et al, 2004). The pattern of results associated with dizocilpine and PCP does not readily fit this description. It remains highly speculative, but the dual suppressive effects on prepulse-elicited reactivity and PPI may stem from NMDA receptor antagonism at multiple sites (Bakshi and Geyer, 1998) by dampening the motor reactivity towards the prepulse stimulus as well as the ability to initiate and/or execute sensorimotor gating.

As a potential animal model of schizophrenia and its pharmacotherapy, it is essential to establish the relative contributions of the diverse pathways whereby the expression of PPI can be modulated. While prepulse-triggered sensorimotor gating and the expression of PPI can be understood as causally related (see Graham, 1975, 1980, 1992), the gating process itself is also under the influence of other factors, for example, alertness, vigilance and arousal. The possibility that a given drug can influence multiple mechanisms in the modulation of PPI expression surely deserves serious consideration. Our emphasis on prepulse processing, and in particular as indexed by prepulse-elicited reactivity here, is not meant to undermine the validity of PPI as an operational measure of sensorimotor gating. Instead, it aims to encourage research in prepulse processing itself and thus to supplement our understanding of the psychophysiology of PPI, as well as of PPI-altering manipulations.

It has often been considered that the disruption of PPI induced by PCP and dizocilpine may be more suited to capture the negative symptoms of schizophrenia, while that induced by apomorphine the positive florid symptoms of the disease (Swerdlow and Geyer, 1998; Swerdlow et al, 1994; Bakshi and Geyer, 1995; Bakshi et al, 1994). The former is more closely associated with a functional hyperdopaminergia centered on the mesolimbic system, and the latter may be partly attributed to NMDA receptor hypofunction in the frontal cortices (see Olney et al, 1999; Lieberman et al, 1997). With respect to our present and previous findings (Yee et al, 2004), it would be interesting to test whether the PPI disruptions seen in positively and negatively symptomatic patients are associated with differential alterations in prepulse-elicited reactivity.

\section{ACKNOWLEDGEMENTS}

The present study was supported by the Swiss Federal Institute of Technology. BK Yee received additional support from the NCCR: Neural Plasticity and Repair, Swiss National Science Foundation. We thank Peter Schmid and Misa Yamanaka, respectively, for their technical and editorial assistance. We remain also indebted to Jeanne von Arx and Pascal Guela for their care of the animals and to Dr Frank Bootz for his veterinary expertise.

\section{REFERENCES}

Bakshi VP, Geyer MA (1995). Antagonism of phencyclidineinduced deficits in prepulse inhibition by the putative atypical antipsychotic olanzapine. Psychopharmacology 122: 198-201.

Bakshi VP, Geyer MA (1998). Multiple limbic regions mediate the disruption of prepulse inhibition produced in rats by the noncompetitive NMDA antagonist dizocilpine. J Neurosci 18: 8394-8401.
Bakshi VP, Swerdlow NR, Geyer MA (1994). Clozapine antagonizes phencyclidine-induced deficits in sensorimotor gating of the startle response. J Pharmacol Exp Ther 271: 787-794.

Braff DL, Geyer MA, Swerdlow NR (2001). Human studies of prepulse inhibition of startle: normal subjects, patient groups, and pharmacological studies. Psychopharmacology 156: 234-258.

Braff DL, Grillon C, Geyer MA (1992). Gating and habituation of startle reflex in schizophrenic patients. Arch Gen Psychiatry 49: 206-215.

Curzon P, Decker MW (1998). Effects of phencyclidine (PCP) and (+)MK-801 on sensorimotor gating in CD-1 mice. Prog Neuropsychopharmacol Biol Psychiatry 22: 129-146.

Dahmen JC, Corr PJ (2004). Prepulse-elicited startle in prepulse inhibition. Biol Psychiatry 55: 98-101.

Davis M, Mansbach RS, Swerdlow NR, Campeau S, Braff DL, Geyer MA (1990). Apomorphine disrupts the inhibition of acoustic startle induced by weak prepulses in rats. Psychopharmacology 102: 1-4.

Geyer MA, Krebs-Thomson K, Braff DL, Swerdlow NR (2001). Pharmacological studies of prepulse inhibition models of sensorimotor gating deficits in schizophrenia: a decade in review. Psychopharmacology 156: 117-154.

Geyer MA, Mcllwain KL, Paylor R (2002). Mouse genetic models for prepulse inhibition: an early review. Mol Psychiatry 7: 1039-1053.

Geyer MA, Swerdlow NR (1998). Measurement of startle response, prepulse inhibition, and habituation. In: Crawley JN, Skolnick P (eds). Current Protocols in Neuroscience, Chapter 8.7, Wiley: New York. pp 1-15.

Graham FK (1975). The more or less startling effects of weak prestimulation. Psychophysiology 12: $238-248$.

Graham FK (1980). Control of blink reflex excitability. In: Thompson RF, Hicks LH, Shvyrkov VB (eds). Neural Mechanisms of Goal-directed Behavior and Learning. Academic Press: New York. pp 511-519.

Graham FK (1992). Attention: the heartbeat, the blink, and the brain. In: Campbell BA, Hayne H, Richardson R (eds). Attention and Information Processing in Infants and Adults: Perspectives from Human and Animal Research. Lawrence Erlbaum Associates: Hillsdale, NJ. pp 3-29.

Hoffman HS, Searle JR (1965). Ascoustic variables in the modification of startle reaction in the rat. J Compar Exp Psychol 60: 53-58.

Kretschmer BD, Koch M (1998). The ventral pallidum mediates disruption of prepulse inhibition of the acoustic startle response induced by dopamine agonists, but not by NMDA antagonists. Brain Res 798: 204-210.

Lieberman JA, Sheitman BB, Kinon BJ (1997). Neurochemical sensitization in the pathophysiology of schizophrenia: deficits and dysfunction in neuronal regulation and plasticity. Neuropsychopharmacology 17: 205-229.

Martinez ZA, Halim ND, Oostwegel JL, Geyer MA, Swerdlow NR (2000). Ontogeny of phencyclidine and apomorphine-induced startle gating deficits in rats. Pharmacol Biochem Behav 65: 449-457.

Olney JW, Newcomer JW, Farber NB (1999). NMDA receptor hypofunction model of schizophrenia. J Psychiatr Res 33: 523-533.

Ralph-Williams RJ, Lehmann-Masten V, Geyer MA (2003). Dopamine D1 rather than D2 receptor agonists disrupt prepulse inhibition of startle in mice. Neuropsychopharmacology 28: 108-118.

Snyder SH (1976). The dopamine hypothesis of schizophrenia: focus on the dopamine receptor. Am J Psychiatry 133: 197-202.

Swerdlow NR, Braff DL, Geyer MA (2000). Animal models of deficient sensorimotor gating: what we know, what we think we know, and what we hope to know soon. Behav Pharmacol 11: 185-204. 


\section{MK-80 I, PCP, and prepulse-elicited reactivity}

BK Yee et al

Swerdlow NR, Braff DL, Taaid N, Geyer MA (1994). Assessing the validity of an animal model of deficient sensorimotor gating in schizophrenic patients. Arch Gen Psychiatry 51: 139-154.

Swerdlow NR, Geyer MA (1998). Using an animal model of deficient sensorimotor gating to study the pathophysiology and new treatments of schizophrenia. Schizophr Bull 24: 285-301.

Swerdlow NR, Paulsen J, Braff DL, Butters N, Geyer MA, Swenson MR (1995). Impaired prepulse inhibition of acoustic and tactile startle response in patients with Huntington's disease. J Neurol Neurosurg Psychiatry 58: 192-200.

Varty GB, Walters N, Cohen-Williams M, Carey GJ (2001). Comparison of apomorphine, amphetamine and dizocilpine disruptions of prepulse inhibition in inbred and outbred mice strains. Eur J Pharmacol 424: 27-36.

Yee BK (2000). Cytotoxic lesion of the medial prefrontal cortex abolishes the partial reinforcement extinction effect, attenuates prepulse inhibition of the acoustic startle reflex and induces transient hyperlocomotion, while sparing spontaneous object recognition memory in the rat. Neuroscience 95: 675-689.

Yee BK, Russig H, Feldon J (2004). Apomorphine-induced prepulse inhibition disruption is associated with a paradoxical enhancement of prepulse stimulus reactivity. Neuropsychopharmacolgy 29: $240-248$.

Zorrilla EP (1997). Multiparous species present problems (and possibilities) to developmentalists. Dev Psychobiol 30: 141-150. 